\title{
Electroacupuncture to Increase Neuronal Stem Cell Growth
}

\author{
Genia Dubrovsky, MD, ${ }_{1}$ Don Ha, LAc, ${ }^{2}$ Anne-Laure Thomas, MS, ${ }^{3}$ Minji Zhu, MS, \\ John Hubacher, MA, ${ }^{5}$ Tatsuo Itoh, $\mathrm{PhD},{ }^{4}$ and James C.Y. Dunn, MD, $\mathrm{PhD}^{1,3}$
}

\begin{abstract}
Background: Neuropathic intestinal disorders continue to pose a significant burden, and current treatment options do not target the underlying cellular deficiencies. The goal of this study is to determine whether acupuncture and electroacupuncture (EA) can affect the growth of neuronal cells.

Methods: Three groups of Lewis rats received 25 minutes of acupuncture twice a week for 10 weeks. The 3 groups of rats received treatment with either sham acupuncture (SA), real acupuncture (RA), or EA. After 10 weeks of treatment, skin and intestinal tissue were collected and analyzed for histology and mRNA expression of neuronal marker genes.

Results: Compared with rats that received SA, rats that received RA and EA showed a significant increase in the mRNA expression levels of multiple neuronal genes in the skin. No significant histologic changes were seen.

Conclusions: Acupuncture and EA result in significant changes in the expression of genes implicated as markers for neural stem cells, neural cell development, and neurons. This may, therefore, provide a novel avenue for developing treatments in patients suffering from intestinal aganglionic and neuropathic diseases.
\end{abstract}

Keywords: electroacupuncture, stem cells, aganglionic diseases, neuropathic diseases

\section{INTRODUCTION}

$\mathbf{H}$ IRSCHSPRUNG DISEASE (HD) is the most common congenital intestinal motility disorder and affects 1 in 5,000 to 10,000 live births. ${ }^{1-4}$ The disease is caused by an absence of enteric ganglia in either a portion of the colon or the entire colon and results in life-threatening intestinal obstruction. ${ }^{1-4}$ A number of other more rare but still significant intestinal diseases can also result from pathology involving the enteric nervous system (ENS) in the pediatric population. These include, but are not limited to, achalasia, gastroparesis, and chronic intestinal pseudo-obstruction (CIPO) ${ }^{4,5}$ Some of these diseases such as HD have effective treatment options; resection of the aganglionic colonic segment is life saving, although significant symptoms of both constipation and incontinence can persist into adulthood. ${ }^{3,6}$ Other diseases such as CIPO have no definitive therapies. ${ }^{2,5}$ Overall, none of the current treatments for these diseases attempts to directly remedy the underlying pathology of the ENS.

One modality that has been studied for its ability to regenerate nerve cells is electroacupuncture (EA). A number of animal studies have shown that EA is successful in promoting the survival of injured neurons in the spinal cord and brain. ${ }^{7-10}$ There is also some evidence that EA can improve symptoms of gastroparesis and improve intestinal

\footnotetext{
${ }^{1}$ Division of Pediatric Surgery, Department of Surgery, David Geffen School of Medicine, University of California Los Angeles, Los Angeles, CA.

${ }^{2}$ Santa Monica Acupuncture Center, Los Angeles, CA.

${ }^{3}$ Division of Pediatric Surgery, Department of Surgery, Stanford University School of Medicine, Stanford, CA.

${ }^{4}$ Department of Electrical Engineering, University of California Los Angeles, Los Angeles, CA.

${ }^{5}$ Pantheon Research, Inc., Culver City, CA.
}

CME available online at www.medicalacupuncture.org/cme Questions on page 22. 
motility. ${ }^{11,12}$ In this study, we aim to determine whether EA can increase the population of nerve cells in the skin and in the intestine of healthy rats.

\section{MATERIALS AND METHODS}

All research in this study involving animals was approved by the Animal Research Committee (Institutional Review Board No. 2006-061-41). Lewis rats (Charles River Laboratories, Wilmington, MA) aged 8-12 weeks and weighing 200-300 g were used. Stainless steel 32 gauge acupuncture needles (Wabbo, San Jacinto, CA) were connected to a Great Wall KWD-808 I Multi-Purpose Health Device to deliver the electricity. A DS1054Z oscilloscope (Rigol, Beaverton, OR) was used for measuring the electrical signal.

\section{Acupuncture}

Rats were anesthetized with inhaled isoflurane and their backs were shaved. In the sham acupuncture (SA) group, 8 acupuncture needles were placed $\sim 5 \mathrm{~mm}$ deep at random locations on each rat's back for 25 minutes (Fig. 1A). In the real acupuncture (RA) group, 8 acupuncture needles were placed $\sim 5 \mathrm{~mm}$ deep at specific acupuncture points on each rat's back for 25 minutes (Fig. 1B). The points used were BL 11 Dazhu (left and right), GB 30 Huantiao (left and right), GV 14 Dazhui, GV 1 Changqiang, GV 9 Zhiyang, and GV 7 Zhongshu. In the EA group, 8 acupuncture nee- dles were placed $\sim 5 \mathrm{~mm}$ deep at the same acupuncture points on each rat's back, and continuous wave electricity was delivered through 3 pairs of needles for 25 minutes (Fig. 1C). The 3 pairs of points used for electricity were BL 11 Dazhu and GB 30 Huantiao (left and right), and GV 14 Dazhui and GV 1 Changqiang. The intensity of the current was adjusted to just barely elicit visible muscle contractions. In each rat, these locations were marked by injecting a small amount of India ink (Fisher Scientific, Pittsburgh, PA) intradermally. The same locations were used for each treatment, for a total of 20 treatments for 10 weeks.

\section{Tissue Collection and Histologic Evaluation}

Rats were euthanized after 10 weeks of treatments. Full thickness sections of skin were cut out at each acupuncture site for each rat. Samples were also taken from each animal's small bowel and colon. Half of the sample was saved for RNA analysis (detailed hereunder), and half was used for histology. The samples were placed into $10 \%$ buffered formalin (Fisher Scientific) overnight. They were then imbedded in paraffin, and the paraffin blocks were cut into $5 \mu \mathrm{m}$ sections to create slides. One set of slides was stained with hematoxylin and eosin (H\&E) and viewed under brightfield microscopy. Another set of unstained slides was prepared for immunofluorescence staining.

Unstained slides were dewaxed with xylene and rehydrated with serial dilutions of ethanol. Next, slides were incubated in a citric buffer (Biogenex, Fremont, CA) for 20
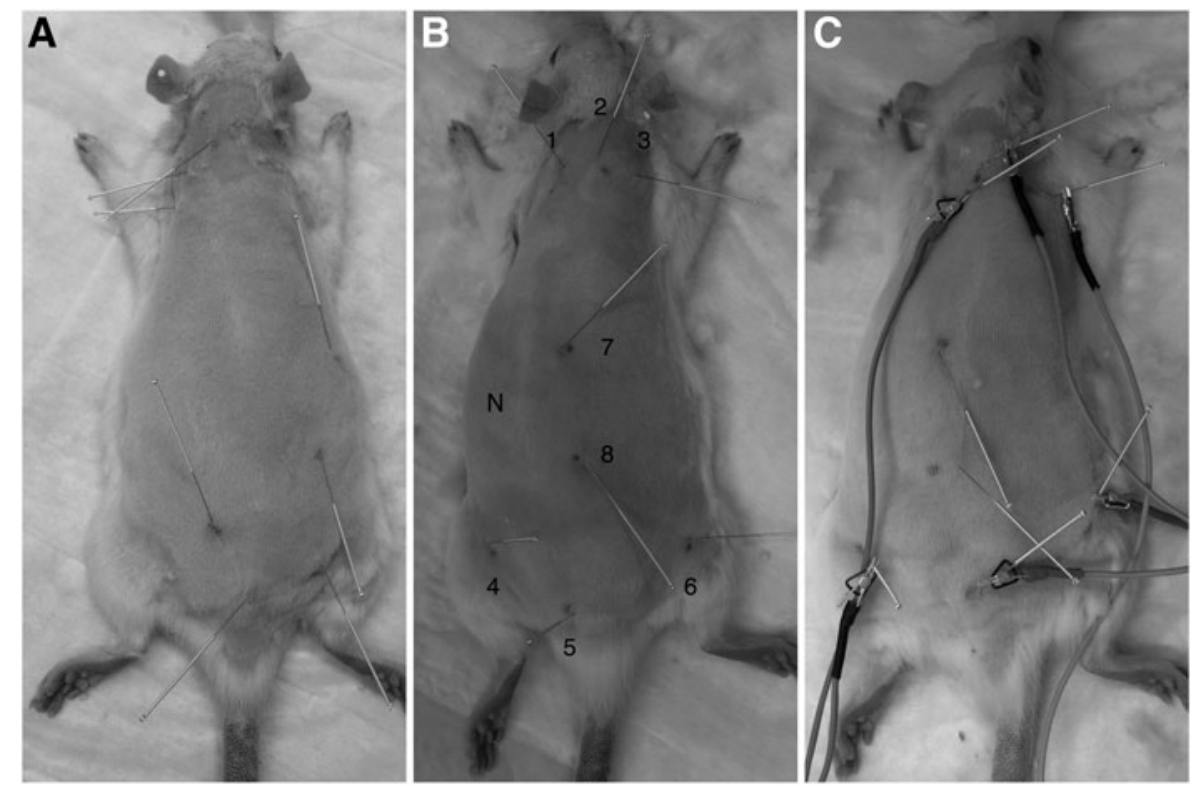

FIG. 1. (A) SA being performed for 25 minutes by placing acupuncture needles at 8 random locations on the rat's back. (B) RA being performed for 25 minutes by placing acupuncture needles at 8 acupuncture points. (C) EA being performed for 25 minutes by placing acupuncture needles at 8 acupuncture locations and delivering electricity through 3 pairs of needles. $1=\mathrm{BL} 11 \mathrm{Dazhu}(\mathrm{left}), 2=\mathrm{GV} 14$ Dazhui, 3=BL 11 Dazhu (right), 4=GB 30 Huantiao (left), 5=GV 1 Changqiang, 6=GB 30 Huantiao (right), $7=$ GV 9 Zhiyang, $8=\mathrm{GV} 7$ Zhongshu, and $N=$ location where normal skin was harvested at time of euthanasia. EA, electroacupuncture; RA, real acupuncture; SA, sham acupuncture. 
minutes at $95^{\circ} \mathrm{C}-100^{\circ} \mathrm{C}$ and allowed to cool for 15 minutes in an over-flowing water bath. Samples on slides were permeabilized in $1 \%$ Triton-X for 5 minutes. After 3 washes with phosphate-buffered saline (PBS), samples were treated with blocking buffer containing $4 \%$ normal goat serum (Vector Labs, Burlingame, CA) and 2\% bovine serum albumin fraction V (Janssen Pharmaceuticalaan, Gent, Belgium) in PBS for 1 hour at room temperature. Samples were then incubated in blocking buffer overnight at $4{ }^{\circ} \mathrm{C}$ with 1:200 of a primary polyclonal antibody against the neural stem cell markers: $\alpha$-Nestin (Abcam, Cambridge, United Kingdom), SOX10 (Abcam), $\beta$-tubulin III (Abcam), S100b (Dako, Glostrup, Denmark), and p75 (Abcam). After 3 washes in PBS, samples were incubated in the dark with a secondary antibody (goat antirabbit Alexa Fluor 488; Invitrogen) diluted at 1:200 in PBS for 1 hour at room temperature. After 3 washes with PBS, Prolong Diamond with DAPI (Invitrogen) was applied to each section and the slides were covered with glass cover slips. Images were acquired with an Olympus IX73 microscope with cellSens software (Olympus, Center Valley, PA).

\section{Quantitative reverse transcription polymerase chain reaction}

Skin samples were cut into pieces with scissors and emulsified with a homogenizer on ice. Total RNA was extracted from the samples using the RNeasy Mini Kit (Qiagen, Hilden, Germany) following the protocol provided from Qiagen. The total amount and the purity of RNA were measured using the absorbance of the sample at 230, 260, and $280 \mathrm{~nm}$. The ABI Prism 7900 Sequence Detection System (Applied Biosystems, Carlsbad, CA) and PCR master mix from Quantitect Probe reverse transcription polymerase chain reaction (RT-PCR) kit
(Qiagen) were used to perform the real-time (RT)-polymerase chain reactions (PCRs). PCR primers and probes for amplification of glyceraldehyde 3-phosphate dehydrogenase (GAPDH, Rn01775763_g1), Nestin (Rn00564396_m1), S100b (Rn04219408_m1), $\beta$-tubulin III (Rn01431594_m1), p75 neurotrophin receptor (Rn00561634_m1), and SOX10 (Rn00569909_m1) were purchased from Taqman (Life Technologies, Carlsbad, CA) and were used according to the protocol provided by the vendor at $1 \times$ final concentration in the PCR mixture. All PCRs were carried out in a $10 \mu \mathrm{L}$ volume in a 384-well RT-PCR plate (Applied Biosystems) with the following: $6.25 \mu \mathrm{L} /$ well of master mix (Qiagen), $0.5 \mu \mathrm{L}$ of each primer $(1 \times), 0.5 \mu \mathrm{L}$ of the probe $(1 \times), 1.625 \mu \mathrm{L}$ of DNase/RNase-free water (Qiagen), and $1.5 \mu \mathrm{L}$ of each RNA sample. Samples were run in duplicate and normalized to the sample of normal skin from each animal group. When this was not possible because of an inadequate sample, the samples were normalized to the normal skin of the SA group.

\section{Statistical Analysis}

All data were recorded as mean \pm standard deviations. Two-tailed unpaired Student's $t$-tests were used for statistical analysis of electrical measurements, immunostaining, and RT-PCR data between groups.

\section{RESULTS}

All rats tolerated their treatments and gained weight over the course of 10 weeks. Measurements of the electrical currents showed that the rats in the EA group received a biphasic impulse with a total frequency of $5 \mathrm{~Hz}$. The positive impulse had a pulse width of $0.6 \mathrm{~ms}$, a voltage of
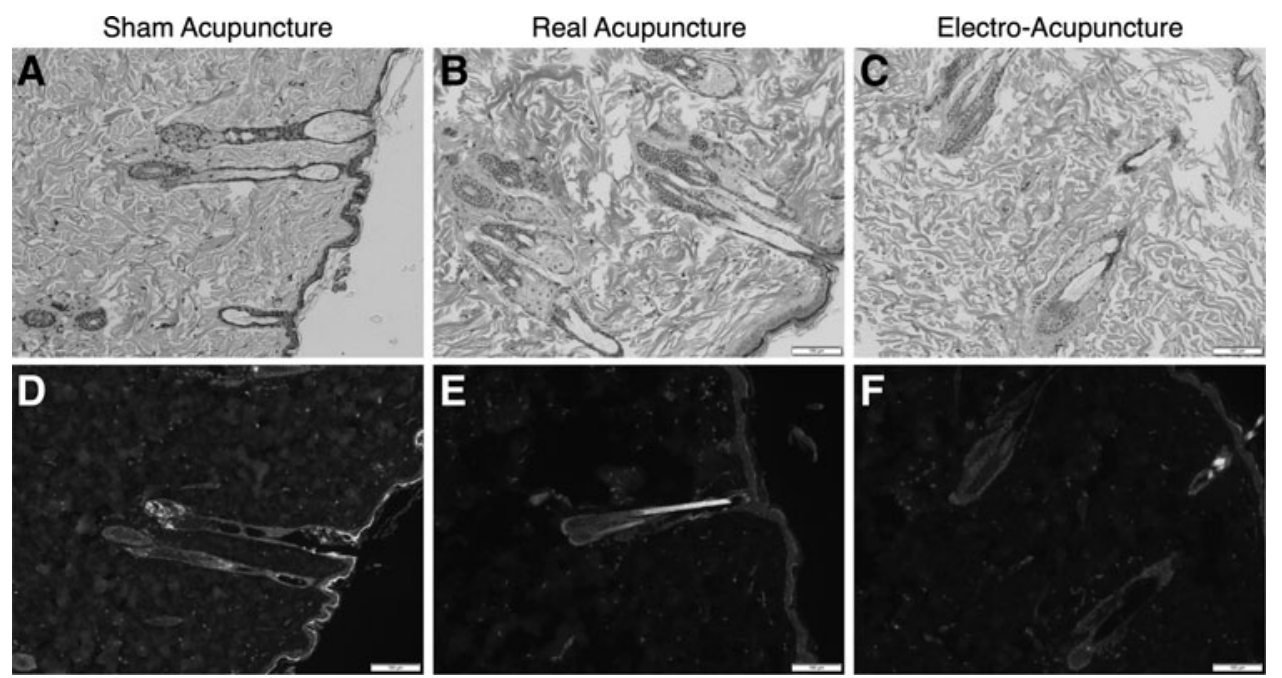

FIG. 2. (A-C) Representative H\&E sections of skin from SA, RA, and EA rats under brightfield microscopy at $100 \times$ magnification (scale bars mark distance of $100 \mu \mathrm{m}$ ). (D-F) Representative sections of skin with immunostaining for Nestin (green) and staining with DAPI (blue) from SA, RA, and EA rats at 100× magnification (scale bars mark distance of $100 \mu \mathrm{m}$ ). H\&E, hematoxylin and eosin. 

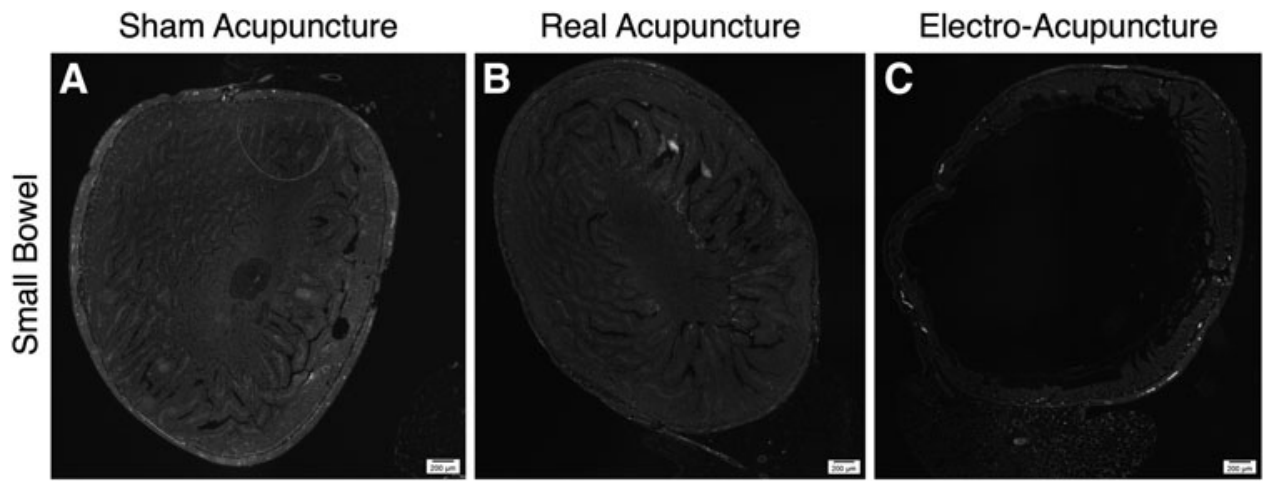

FIG. 3. Representative sections of small bowel with immunostaining for Nestin (green) from (A) SA, (B) RA, and (C) EA rats (scale bars mark distance of $200 \mu \mathrm{m})$.

$3.4 \pm 1.5 \mathrm{~V}$, and a current of $1.6 \pm 0.6 \mathrm{~mA}$. The negative impulse had a pulse width of $6.0 \mathrm{~ms}$, a voltage of $0.6 \pm 0.4 \mathrm{~V}$, and a current of $1.1 \pm 0.3 \mathrm{~mA}$. There was no significant difference in the current across the 3 different channels.

\section{Histologic Evaluation}

Figure 2 shows representative sections from the skin after $H \& E$ staining and after immunostaining for Nestin. There was no significant difference in the amount of staining seen for Nestin, S100b, $\beta$-tubulin III, SOX10, or p75 in the skin among the 3 different groups. Figures 3 and 4 show the representative sections from small bowel and colon stained for Nestin. Again, there was no significant difference seen in the staining among the 3 different groups for any of the 5 neuronal markers.

\section{Quantitative RT-PCR}

RT-PCR showed significantly higher mRNA levels of Nestin, SOX10, and p75 in the skin of the upper back than in normal skin after SA $(P<0.05)$. There were significantly lower levels of S100b expression in the skin of the lower back and in the small bowel than in normal skin after SA.
The RA group had significantly higher levels of expression of SOX10, S100b, and p75 in the upper back skin than those in the normal skin. RA also showed a higher $\mathrm{p} 75$ expression in the small bowel than in the normal skin. The EA group had significantly higher levels of Nestin, S100b, and p75 in the skin of the upper back than those in the normal skin. Levels of Nestin were significantly lower in the EA group in the small bowel than in the normal skin. Finally, the expression levels in the RA group were significantly greater than in the SA group for SOX10 and p75 in the upper back skin, and for S100b in the upper and lower back skin. The expression levels in the EA group were significantly greater than in the SA group for S100b in the upper and lower back skin. Expression of p75 was significantly higher in the RA group than in the EA group in the skin of the upper back. The expression of p75 in the small bowel of the RA group was also greater than in either the SA or EA group (Fig. 5).

\section{DISCUSSION}

We found that both RA and EA result in significant increases in the expression of the neuronal genes Nestin,
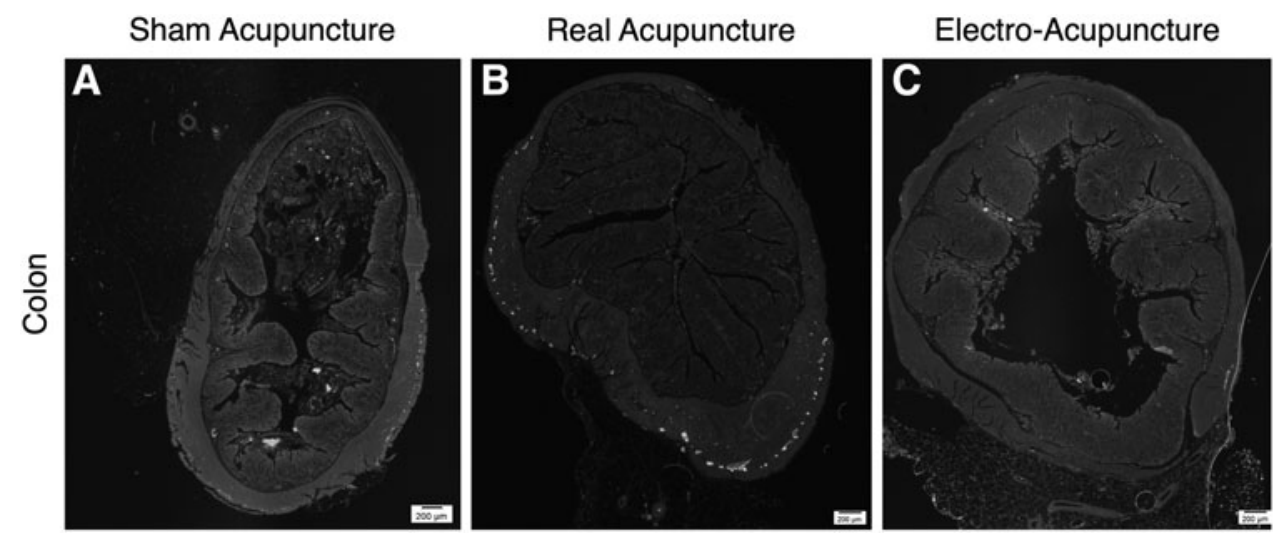

FIG. 4. Representative sections of colon with immunostaining for Nestin (green) at from (A) SA, (B) RA, and (C) EA rats (scale bars mark distance of $200 \mu \mathrm{m}$ ). 
A

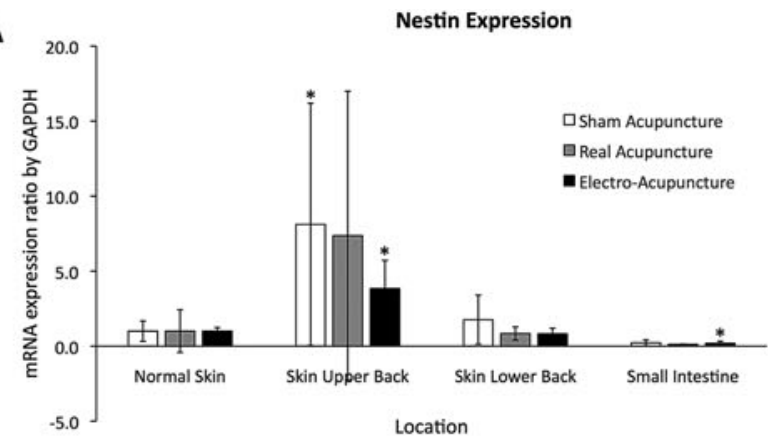

B

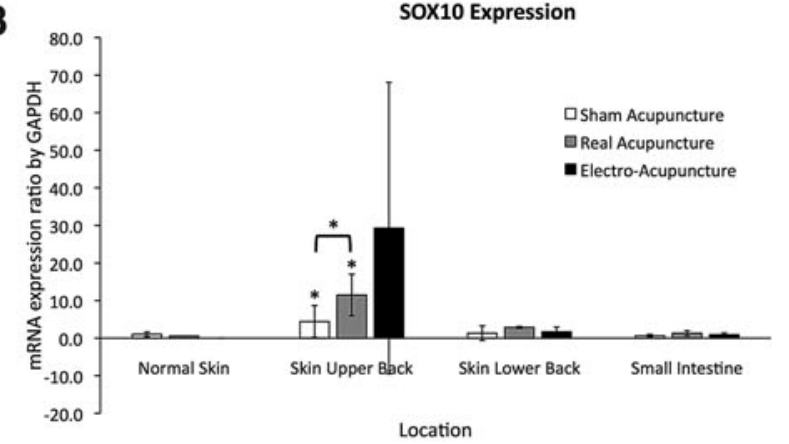

C

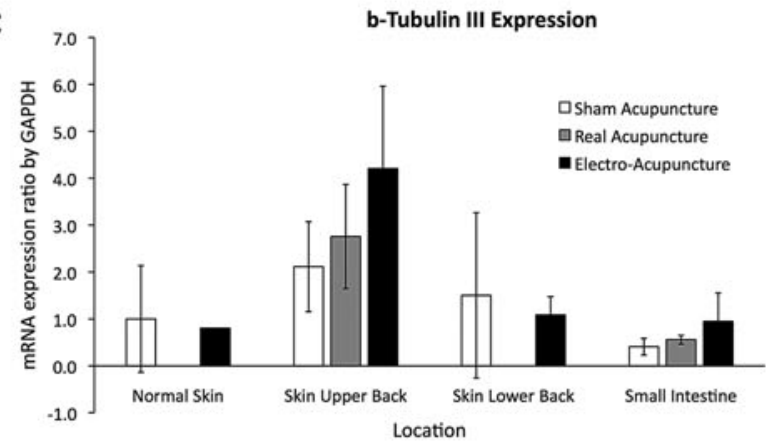

D

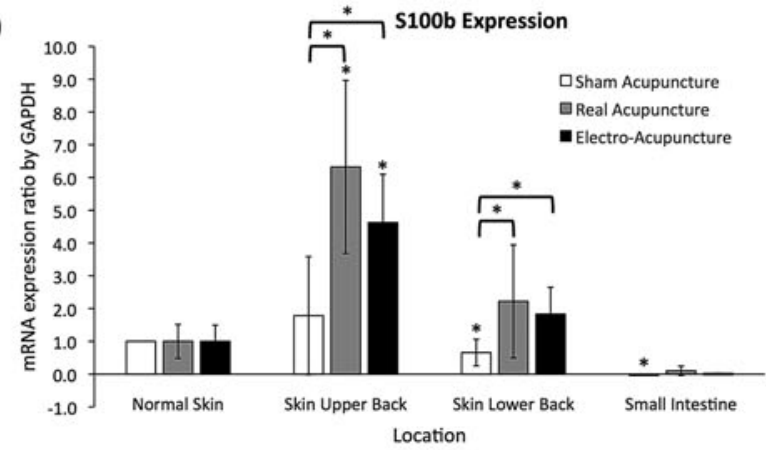

E

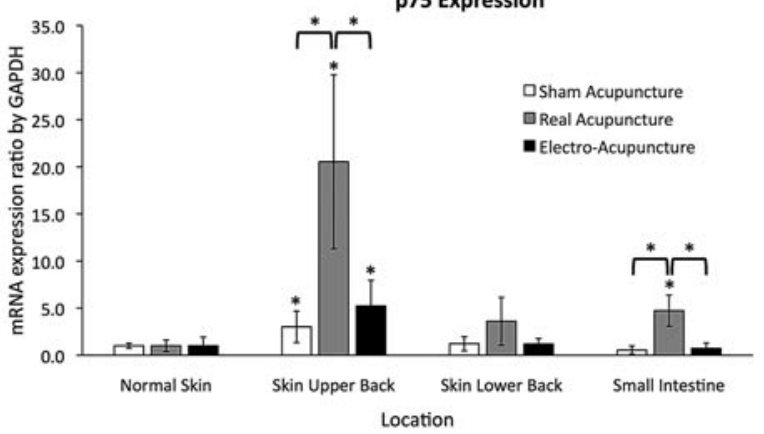

SOX10, $\beta$-tubulin III, S100b, and p75 in the skin, but not in the intestine. Although SA also results in increased expression of Nestin, SOX10, S100b, and p75, it is never significantly greater than the results from RA and EA. In contrast, RA results in greater expression of SOX10, S100b, and p75 than SA. EA results in significantly greater expression of S100b, and also a trend toward greater expression of SOX10 and $\beta$-tubulin III, although those differences are not statistically significant. When comparing RA and EA, it is difficult to tell based on our results which one has the greatest effect on neuronal gene expression. RA has a significantly greater effect on $\mathrm{p} 75$ expression, whereas the other genes are similarly affected by both modalities.

Nestin is a type IV intermediate filament and thus a component of the cytoskeleton [13]. It is used as a marker for neuronal progenitor cells both in the central and peripheral nervous systems, including in the skin and intestine. ${ }^{13}$ SOX10 is a transcription factor important in the development of the ENS and the peripheral nervous system. ${ }^{14}$ Mutations in this gene can result in HD. ${ }^{14} \beta$-Tubulin III is another cytoskeletal protein, and is a building block of microtubules. ${ }^{15}$ This protein is normally limited to the central and peripheral nervous systems, and to the testes. ${ }^{15}$ $\mathrm{S} 100 \mathrm{~b}$ is a calcium-binding protein found in several cell lines including neural progenitor cells, and some other neuronal cells. ${ }^{16}$ It promotes neuronal survival and regeneration. ${ }^{16}$ p75 is a receptor involved in cell signaling, differentiation, and death. ${ }^{17}$ It is expressed in neural crest cells, which give rise to peripheral and enteric neurons, and glial cells among others. ${ }^{17}$ Taken together, the increased expression of these genes indicates that RA and EA likely result in the growth of neuronal progenitor cells in the skin.

Several past studies have indicated similar results of increased neuronal progenitor cells as a result of EA. In a rat spinal cord injury model, 1 week of EA resulted in increased Nestin expression in the injured spinal cord segment. ${ }^{7}$ In a rat stroke model, 3 days of EA resulted in increased Nestinpositive cells in the brain, and a decreased infarct volume. ${ }^{10}$ Importantly, these studies show that it is not only possible to recruit neuronal progenitor cells, but that they can also be localized to the tissue of interest. In this study, although we did see an increase in neuronal markers in the skin, we did not see a corresponding increase in the small bowel and colon. One solution may be to use different acupuncture points. In fact, past studies have shown that intestinal motility in a mouse model can be increased through EA stimulation at

FIG. 5. Ratio of expression of (A) Nestin, (B) SOX10, (C) Btubulin III, (D) S100b, and (E) p75 to GAPDH in SA, RA, and EA rats. All values are normalized and expressed relative to GAPDH and also relative to levels in the normal skin of the animals in each acupuncture group. *Indicates $P$-value $<0.05$. Figure 1 shows the locations where the skin was harvested. Points 1-6 are all different acupuncture points, and "normal skin" (N) was taken from an area away from any needles. 
Shangjuxu. ${ }^{12}$ Similarly, a human study using points Shangjuxu, Neiguan, Sanyanglou, Zusanli, Xiajuxu, and Taibai showed improved gastric emptying in critically ill adults being treated with metoclopramide. ${ }^{11}$

Another method to localize the neuronal progenitor cells to the tissue of interest may be through harvesting and injecting them. A previous study used a transected spinal cord rat model to study EA. ${ }^{9}$ They found that injecting mesenchymal stem cells at the site of injury combined with EA resulted in better outcomes than simply injection of mesenchymal stem cells or EA alone. ${ }^{9}$ Specifically, EA promoted the differentiation of the injected mesenchymal stem cells, and this led to a greater regeneration of nerve fibers and an improved recovery of movement. ${ }^{9}$ Thus the increase of neuronal progenitor cells in the skin alone may be of benefit if these cells can be harvested and then introduced into a target tissue. Injection of stem cells for the treatment of HD is already an active and promising area of research and EA may provide an added advantage in generating the necessary stem cells. ${ }^{18,19}$

One limitation of this study is that we did not see consistent correlation between our RT-PCR results and the immunostaining. Although there was a significant increase in the mRNA expression of several neuronal markers, we were not able to identify these changes visually. It is possible that not all the RNA was directly translated to the protein level. The immunostaining may also not have been as sensitive in detecting changes as the RT-PCR. Another limitation is that although we have shown that RA and EA result in an increase in neuronal markers, we have not yet shown that this results in increased function. Further studies will be needed to see whether this population of neuronal cells may be used for therapeutic purposes.

\section{CONCLUSIONS}

We show that RA and EA result in an increased expression of neuronal stem cell genes in the skin as compared with SA. Although these same changes are not seen in the intestine, it may be possible to harvest the skin neuronal stem cells for therapeutic use. This may provide future novel therapies in the treatment of a wide range of neuropathic intestinal diseases in the pediatric population.

\section{ACKNOWLEDGMENTS}

We thank the Translational Pathology Core Laboratory at UCLA for its help in preparing histologic samples.

\section{AUTHOR CONTRIBUTIONS}

J.C.Y.D., D.H., and T.I. conceived and designed the study. G.D., D.H., A-L.T., M.Z., and J.H. acquired data.
J.C.Y.D. and G.D. analyzed and interpreted data. G.D. drafted the article. J.C.Y.D. and G.D critically revised the article.

\section{AUTHOR DISCLOSURE STATEMENT}

No competing financial interests exist.

\section{FUNDING INFORMATION}

This research was funded by the Adolph Coors Foundation.

\section{REFERENCES}

1. Langer JC. Hirschsprung disease. Curr Opin Pediatr. 2013; 25(3):368-374.

2. Koppen IJN, Benninga MA, Singendonk MMJ. Motility disorders in infants. Early Hum Dev. 2017;114:1-6.

3. Goldstein AM, Thapar N, Karunaratne TB, De Giorgio R. Clinical aspects of neurointestinal disease: Pathophysiology, diagnosis, and treatment. Dev Biol. 2016;417(2):217-228.

4. Chumpitazi B, Nurko S. Pediatric gastrointestinal motility disorders: Challenges and a clinical update. Gastroenterol Hepatol (N Y). 2008;4(2):140-148.

5. Westfal ML, Goldstein AM. Pediatric enteric neuropathies: Diagnosis and current management. Curr Opin Pediatr. 2017; 29(3):347-353.

6. Rintala RJ, Pakarinen MP. Outcome of anorectal malformations and Hirschsprung's disease beyond childhood. Semin Pediatr Surg. 2010;19(2):160-167.

7. Zhu Y, Wu Y, Zhang R. Electro-acupuncture promotes the proliferation of neural stem cells and the survival of neurons by downregulating miR-449a in rat with spinal cord injury. EXCLI J. 2017;16:363-374.

8. Zhang YT, Jin $\mathrm{H}$, Wang $\mathrm{JH}$, et al. Tail nerve electrical stimulation and electro-acupuncture can protect spinal motor neurons and alleviate muscle atrophy after spinal cord transection in rats. Neural Plast. 2017;2017:7351238.

9. Yan Q, Ruan JW, Ding Y, Li WJ, Li Y, Zeng YS. Electroacupuncture promotes differentiation of mesenchymal stem cells, regeneration of nerve fibers and partial functional recovery after spinal cord injury. Exp Toxicol Pathol. 2011; 63(1-2):151-156.

10. Chen B, Tao J, Lin Y, Lin R, Liu W, Chen L. Electroacupuncture exerts beneficial effects against cerebral ischemia and promotes the proliferation of neural progenitor cells in the cortical peri-infarct area through the $\mathrm{Wnt} / \beta$ catenin signaling pathway. Int J Mol Med. 2015;36(5): 1215-1222.

11. Kao ML, Chen YL, Lee SC, Huang SY, Lin PY. Electroacupuncture improves gastric emptying in critically ill neurosurgical patients: A pilot study. Evid Based Complement Alternat Med. 2017;2017:1892161.

12. Liang $\mathrm{C}$, Wang $\mathrm{K}, \mathrm{Xu} \mathrm{B}, \mathrm{Yu} \mathrm{Z}$. Electroacupuncture at acupoint ST 37(Shangjuxu) improves function of the enteric 
nervous system in a novel mouse constipation model. $B M C$ Complement Altern Med. 2016;16(1):392.

13. Bernal A, Arranz L. Nestin-expressing progenitor cells: Function, identity and therapeutic implications. Cell Mol Life Sci. 2018.

14. Bondurand N, Sham MH. The role of SOX10 during enteric nervous system development. Dev Biol. 2013;382(1):330-343.

15. Mariani M, Karki R, Spennato M, et al. Class III $\beta$-tubulin in normal and cancer tissues. Gene. 2015;563(2):109-114.

16. Donato R, Sorci G, Riuzzi F, et al. S100B's double life: Intracellular regulator and extracellular signal. Biochim Biophys Acta. 2009;1793(6):1008-1022.

17. Pincelli C. p75 Neurotrophin receptor in the skin: Beyond its neurotrophic function. Front Med (Lausanne). 2017;4:22.

18. Wagner JP, Sullins VF, Dunn JC. Transplanted skin-derived precursor stem cells generate enteric ganglion-like structures in vivo. J Pediatr Surg. 2014;49(8):1319-1324; discussion 1324-1325.

19. Fattahi F, Steinbeck JA, Kriks S, et al. Deriving human ENS lineages for cell therapy and drug discovery in Hirschsprung disease. Nature. 2016;531(7592):105-109.

Address correspondence to:

James C.Y. Dunn, MD, PhD Division of Pediatric Surgery Department of Surgery Stanford University School of Medicine 300 Pasteur Drive, Alway M116 Stanford, CA 94305

E-mail: jdunn2@stanford.edu

\section{To receive CME credit, you must complete the quiz online at: www.medicalacupuncture.org/cme}

\section{CME Quiz Questions}

Article learning objectives: After studying this article, participants should be able to distinguish some of the relevant research investigating acupuncture effects on neuronal stem cell growth; identify some methods of current research on acupuncture and neuronal stem cell growth; and assess some of the peer reviewed scientific literature on acupuncture and neuronal stem cell growth.

Publication date: February 3, 2020

Expiration date: February 28, 2021

\section{Disclosure Information:}

Authors have nothing to disclose.

Richard C. Niemtzow, MD, PhD, MPH, Editor-in-Chief, has nothing to disclose.

\section{Questions:}

1. Please identify the correct statement:

a. In this paper the authors present a randomized clinical trial of acupuncture for Hirschsprung disease (HD).

b. In this paper the authors review clinical studies on acupuncture's effectiveness for several diseases of the enteric nervous system including achalasia, gastroparesis, and chronic intestinal pseudo-obstruction (CIPO).

c. In this paper the authors utilize a rat model of Hirschsprung disease to examine the effectiveness of acupuncture for this congenital intestinal motility disorder.

d. In this paper the authors utilize a rat model of Hirschsprung disease to examine the ability of acupuncture to regenerate nerve cells. e. In this paper the authors use Lewis rats to explore the ability of acupuncture to effect the expression of mRNA of neuronal marker genes.

2. Please identify the incorrect statement:

a. The purpose of this study was to determine if electroacupuncture can increase the population of nerve cells in the skin and intestine of healthy rats.

b. Using a rat model, needle placement $5 \mathrm{~mm}$ deep at random points on the back was utilized as a sham acupuncture control.

c. "Real acupuncture" (RA) was designated as needle placement $5 \mathrm{~mm}$ deep at BL 11, GB30, GV14, GV1, GV9, and GV7.

d. "Electroacupuncture" (EA) was designated as application of a biphasic impulse with a total fre- 
quency of $5 \mathrm{~Hz}$ to 3 pairs of the needles at the same points as the RA group.

e. The authors give a detailed explanation of the rationale for their choice of acupuncture points based on combination of: prior research, clinical experience, Chinese medical theory of points with a described action on the intestines.

3. The researchers in this study found by applying real acupuncture they significantly increased expression of neuronal genes Nestin, SOX10 and p 75 in the (choose one):
a. intestine
b. nervous system
c. skin
d. muscle
e. fascia

4. According to this research article, all of the following statements are true about research on electroacupuncture EXCEPT (choose one):

a. EA promoted the differentiation of injected mesenchymal stem cells in a transected spinal cord rat model.

b. EA had a significantly greater effect than RA on neuronal gene mRNA expression in the authors' study on healthy Lewis rats. c. EA led to a greater regeneration of nerve fibers in a transected spinal cord rat model.

d. EA increased expression of the neuronal genes in the skin but not the intestine.

e. EA and RA both resulted in significant increases in the expression of neuronal genes Nestin, SOX10, and $\mathrm{p} 75$.

5. Please identify the incorrect statement about this study:

a. There was not a correlation seen between the reverse transcriptase-polymerase chain reaction results and the immunostaining of skin tissue.

b. RT-PCR gave distinct evidence of increased expression of neuronal markers however it did not demonstrate visually an increased presence of neuronal cells.

c. Nestin is a marker for neuronal progenitor cells in the central and peripheral nervous system, including in the skin and intestine.

d. The authors suggest that EA may have a role in generating stem cells but that further research is needed to confirm this.

e. This study provides evidence that electroacupuncture is ready for immediate incorporation into therapy for Hirschsprung disease.

\section{Continuing Medical Education - Journal Based CME Objectives:}

Articles in Medical Acupuncture will focus on acupuncture research through controlled studies (comparative effectiveness or randomized trials); provide systematic reviews and meta-analysis of existing systematic reviews of acupuncture research and provide basic education on how to perform various types and styles of acupuncture. Participants in this journal-based CME activity should be able to demonstrate increased understanding of the material specific to the article featured and be able to apply relevant information to clinical practice.

\section{CME Credit}

You may earn CME credit by reading the CME-designated article in this issue of Medical Acupuncture and taking the quiz online. A score of $75 \%$ is required to receive CME credit. To complete the CME quiz online, go to http://www .medicalacupuncture.org/cme - AAMA members will need to login to their member account. Non-members have the opportunity to participate for a small fee.

Accreditation: The American Academy of Medical Acupuncture is accredited by the Accreditation Council for Continuing Medical Education (ACCME).

Designation: The AAMA designates this journal-based CME activity for a maximum of 1 AMA PRA Category 1 Credit $^{\mathrm{TM}}$. Physicians should claim only the credit commensurate with the extent of their participation in the activity. 